DOI: https://doi.org/10.11144/Javeriana.upsy17-3.pofe

\title{
Protocolo de observación de la función de explicación (PROFE): un instrumento para operacionalizar la transmisión de contenidos por parte del profesorado*
}

Observation Protocol of the Explanation Function (OPEF): An Instrument to Operationalize the Transmission of Contents by Teachers

\author{
Ma África Borges del RosAl $^{\mathrm{a}}$ \\ Universidad de La Laguna, España \\ ORCID: http://orcid.org/0000-0001-8267-4401 \\ Cristina Falcón Muñoz de Bustillo \\ Universidad de Granada, España \\ ORCID: http://orcid.org/0000-0001-5091-1230
}

\footnotetext{
a Autora de correspondencia. Correo electrónico: aborges@ull.es
}

Para citar este artículo: Borges, M. A. \& Falcón, C. (2018). Protocolo de observación de la función de explicación (PROFE): un instrumento para operacionalizar la transmisión de contenidos por parte del profesorado. Universitas Psychologica, 17(3), 1-12. https://doi.org/10.11144/Javeriana.upsy17-3. pofe

\section{RESUMEN}

La calidad de la docencia universitaria es fundamental dentro del proceso educativo, lo que exige la evaluación del profesorado, prefiriéndose la metodología observacional cuando se evalúa la conducta de este. El objetivo de este estudio consistió en el análisis pormenorizado de la función docente de explicación, de especial relevancia dentro el escenario educativo, lo que hace importante su análisis. Para ello, se utilizó el instrumento observacional PROFE, para medir los recursos y estrategias utilizados durante la explicación. Se ha puesto a prueba la bondad psicométrica del instrumento mediante fiabilidad, homogeneidad y se ha optimizado el número de sesiones y el tiempo, para posteriormente analizar la conducta de cinco profesores de la Universidad de La Laguna. Palabras clave

metodología observacional; explicación docente; modelo de funciones docentes; Psicología Educativa; bondad psicométrica.

\begin{abstract}
The quality of university teaching is a very important aspect inside the educational process. This aspect makes it necessary the evaluation of teaching professionals. The observational methodology is the one used for evaluation. The objective of this study is the detailed analysis of one teaching function, the explanation. It is a function of particular relevance in the educational setting, so it is very important to analyze it. For that, the instrument PROFE was utilized. It allows the measurement of the resources and strategies used during the explanation. The instrument psychometric goodness has been tested calculating reliability and homogeneity. The number of sessions and time have been optimized. For later the behavior of five professors of La Laguna University has been analyzed.
\end{abstract}


Ma África Borges del Rosal, Cristina Falcón Muñoz de Bustillo.

Keywords

observational methodology; teacher explanation; teaching function model; Educational Psychology; psychometric goodness of fit.

La educación es fundamental para el desarrollo de la humanidad, ya que supone la transmisión de valores, contenidos y múltiples aspectos de vital importancia para el desarrollo de en sí de una sociedad.

Para que los sistemas educativos funcionen y desarrollen su misión, es decisiva la valoración de la calidad educativa. La introducción de España en el Espacio Europeo de Educación Superior, además de proponer un nuevo marco normativo, hace todavía más presente su importancia para alcanzar mayores niveles de excelencia que influyan de forma positiva en los distintos aspectos de la sociedad (Ley 6/2001, 2001).

El logro de esta calidad educativa, hace necesario que se realicen evaluaciones diversas, siendo este uno de los temas de mayor desarrollo e importancia dentro de este marco (Román \& Murillo, 2013). Dada la complejidad que supone todo el proceso educativo, su evaluación es multimodal, debido a que abarca, desde una perspectiva más global, sistemas educativos, instituciones, centros o programas educativos, llegando en lo más concreto a aspectos tales como el aprendizaje de los alumnos o la actividad docente del profesorado (Mateo \& Martínez, 2008).

Dentro de las diversas facetas educativas que pueden ser evaluadas, la del profesorado es una de las más relevantes, ya que constituye una figura fundamental dentro del escenario educativo. Sus comportamientos son objeto de estudio e investigación, dada la relevancia que estos tienen en la transmisión de contenidos y, en definitiva, en el desarrollo de una enseñanza eficaz. Es indudable que una educación de calidad, pasa por un profesorado de calidad (Galán, González-Galán, \& Rodríguez-Patrón, 2014).

Por ello, la evaluación del profesorado alcanza un papel fundamental, el cual se lleva a cabo desde distintas perspectivas: pruebas estandarizadas evaluando los conocimientos del profesor; encuesta a docentes y alumnado; observaciones en clase; portafolios y la estimación de la contribución del profesor, al rendimiento académico de su alumnado (Martínez, 2013). La observación en el aula, aporta conocimiento sobre lo que ahí ocurre, permitiendo analizar con mayor profundidad las interacciones que se producen en el día a día (Mayorga \& López, 2005), que a su vez permite identificar áreas de mejora (Pianta \& Hamre, 2009).

La mayor relevancia que ha cobrado la observación para evaluar conductas en los últimos años, se manifiesta en el desarrollo de instrumentos de observación en el aula (Borges \& Díaz, 2013; Borges, Falcón, \& Díaz, 2016; Cameron, Connor \& Morrison, 2005; Díaz, Borges, Valadez \& Zambrano, 2014; Good, Mulryan, \& McCaslin, 2006), asî como su aplicación a aspectos diversos. Tales como buenas prácticas docentes (Borges et al., 2016; Díaz, Borges, Valadez \& Zambrano, 2015a), desempeño del profesorado con alumnado de necesidades especiales (RodríguezDorta \& Borges, 2017), su utilidad para medir el Burnout (Díaz, Borges \& Valadez, 2013), así como el estudio de determinadas problemáticas metodológicas (Rodríguez-Dorta \& Borges, 2015; Rodríguez-Dorta \& Borges, 2016; Rodríguez-Naveiras \& Borges, 2015), demostrando la validez cruzada del instrumento observacional para medir la conducta del profesorado en sistemas educativos diferentes (Díaz, Borges, Valadez \& Zambrano, 2015b).

El comportamiento del profesorado, mientras imparte docencia, responde una serie de funciones, como queda recogido en el Modelo de Funciones Docentes (Hernández-Jorge, 2005), entre ellas se destaca la explicación, que forma parte de la función de comunicabilidad docente. Se trata de una conducta fundamental para el profesorado, que supone transmitir la información con claridad, estructuración, de forma que se pueda hacer la enseñanza comprensible (Hernández-Jorge, 2005). La transmisión de contenidos se realiza a partir de una serie de estrategias explicadas por la Psicología Educativa, que ayudan a acceder a la información, suscitar esquemas previos del 
alumnado, estructurar, organizar y aplicar la información (Hernández, 1991; Hernández \& García, 1995).

Jiménez y Hernández-Jorge (2001) clasifican las distintas estrategias en: externas a la información y basadas en la información. Dividiendo estas últimas, a su vez, en estrategias de accesibilidad, estructuración, globalidad informativa, enriquecimiento y efectividad. Las estrategias de globalidad informativa, se subdividen en estrategias de estructuración, ensamblaje y esquemas previos. Las de ensamblaje se dividen en esquemas generales y de sintonización específica de dominio afín (sinónimos, plurivías o plurisituaciones y analogías o metáforas) y de dominio pertinente (explicitación o explicación hacia atrás, ejemplificación o lenguaje familiar).

El estudio de Aciego de Mendoza, Domínguez y Martín (2002) se preguntaba al profesorado sobre cuáles estrategias utiliza con mayor frecuencia en el proceso instruccional. En función de ello, las distintas estrategias quedaron agrupadas en cuatro categorías, basadas en una adaptación del modelo teórico de estrategias docentes (Hernández, 1997): estrategias expositivas conclusivas (exposición de contenidos, uso de nuevas tecnologías, entrega de apuntes elaborados o proporcionar referencias bibliográficas), estrategias expositivas suscitadoras (conexión del contenido con los intereses, experiencias previas o conocimientos del alumnado), estrategias interactivas (participación del alumnadodebates, dinámicas de grupo, etc.) y estrategias de descubrimiento (comentario de texto, resolución de problemas, trabajo de campo, etc.). Las más utilizadas fueron éstas últimas, en primer lugar, y las interactivas, en segundo (Aciego de Mendoza et al., 2002). Posteriormente, Hernández-Jorge (2005) clasifica las diferentes estrategias empleadas en el desempeño docente, relacionadas con la explicación, en cuatro categorías (véase tabla 1).

Fombona y Pascual (2011) exponen, como estrategia o recurso, la importancia de las TIC en la docencia universitaria, pues estas herramientas (ordenadores, Internet, redes satelitales, bases de datos, plataformas de enseñanza virtuales, etc.) brindan la oportunidad de crear procesos alternativos y creativos de aprendizaje, donde el profesor adquiere un rol diferente y los estudiantes juegan un papel más activo.

\section{Tabla 1}

Estrategias relacionadas con la explicación (Hernández-Jorge, 2005)

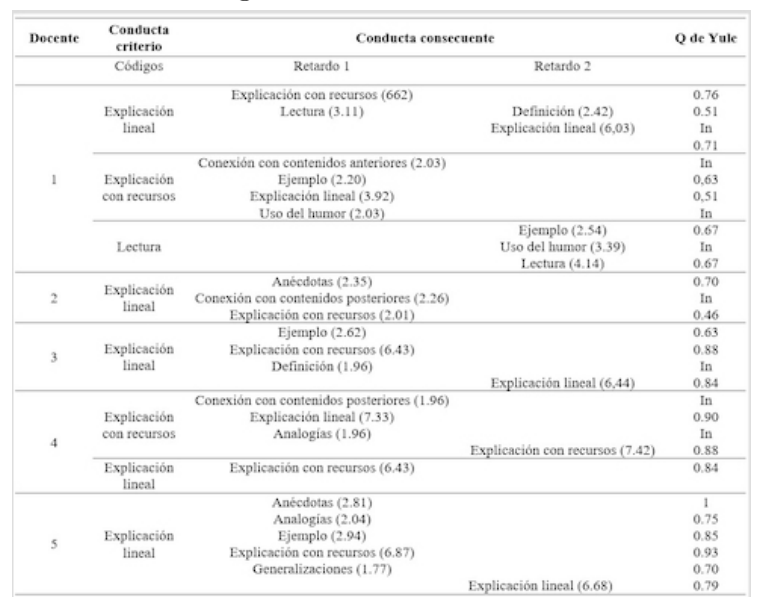

Para poder determinar la calidad del profesorado, es imprescindible operacionalizar su desempeño profesional a través de diferentes instrumentos de medida como: pruebas estandarizadas, encuestas, entrevistas a docentes y alumnado, portafolio, modelos de valor agregado y observaciones en clase (Martínez, 2013). Entre todos los métodos, uno de los mejores procedimientos para estudiar el comportamiento humano en situaciones naturales, lo constituye la metodología observacional (Blanco, Sastre \& Escolano, 2010).

En el contexto de la docencia universitaria, y basado en el Modelo de Funciones Docentes (Hernández-Jorge, 2005), se ha desarrollado el Protocolo de Observación de Funciones Docentes en Universidad, PROFUNDO-UNI (Díaz, 2014), que permite medir tanto las conductas, como los patrones comportamentales de profesorado y alumnado, dentro del aula.

El objetivo de la presente investigación, es la evaluación de la explicación del profesorado universitario a través del instrumento 
observacional, PROFE diseñado para analizar con detalle la conducta: Explicación.

\section{Método}

Se emplea metodología observacional, con diseño nomotético, multidimensional y de seguimiento (Anguera, Blanco \& Losada, 2001).

\section{Participantes}

Han participado cinco docentes, de los cuales tres eran varones. El muestreo ha sido intencional, seleccionándolos por pertenecer a las cinco áreas de conocimiento: artes y humanidades; ciencias; ciencias de la salud; ciencias sociales e ingeniería y arquitectura, de la Universidad de La Laguna. Con edades comprendidas entre los 40 y 60 años, habiendo solicitado su consentimiento informado y el de su alumnado para la grabación de las clases. La observación la han realizado dos observadoras, una doctora en Psicología, de 36 años y la otra estudiante de Psicología, de 23 años.

\section{Instrumentos}

Se ha empleado el Protocolo de Observación de la Función de Explicación (Falcón, 2015), que se muestra en la tabla 2, un instrumento de observación realizado ad hoc, que permite operacionalizar la forma en que el profesorado transmite conocimientos. Dicho instrumento combina un sistema de categorías con formato de campo, siendo cada categoría exhaustiva y mutuamente excluyente. A su vez, los distintos códigos se agrupan formando criterios creados en base al estudio empírico (Anguera \& Hernández-Mendo, 2013). Está conformado por cuatro criterios: Organización del Contenido; Relaciones con los Contenidos; Contraste estimular e Instrumental, desplegado en un total de 21 códigos directamente observables.

Dentro del criterio Organización del Contenido, se encuentran aquellas estrategias destinadas a estructurar e integrar la información, recogiendo las que tienen el objetivo de organizar y clasificar la información, permitiendo así establecer relaciones entre las distintas partes y la totalidad de la información, además de distinguir la información importante de la complementaria (García, 1998). El criterio Relaciones con los Contenidos está formado por todas aquellas estrategias relacionadas con los contenidos teóricos que el docente quiere transmitir; son las estrategias de explicación, de dar significado a la información, hacerla comprensible, de elaborar y producir la información. Las estrategias de explicación (explicación lineal, explicación con recursos, definición, lectura, dictado y repetición), se basan exclusivamente en la transmisión de contenidos, mientras que las de dar significado a la información y hacerla comprensible (uso de sinónimos y analogías), se basan en la utilización de organizadores previos (Ausubel, 1968)., los cuales, tienen el propósito de establecer una conexión entre lo que el alumno sabe y lo que necesita saber. Las estrategias de elaborar y producir la información (generalizaciones), se emplean con el objetivo de que el alumnado sea capaz de generar nuevos conocimientos. Con respecto al criterio Contraste Estimular, se incluyen todas aquellas estrategias que se basan en la introducción de cambios para captar su atención, consiguiendo aumentar su motivación. Dicha estrategia, se basa en el principio gestáltico figura/fondo (HernándezJorge, 2005). Por último, el Criterio Instrumental hace referencia a aquellas conductas que no forman parte de la transmisión de contenidos, pero que se recogen en el instrumento para mantener el flujo continuo de conductas. 


\section{Tabla 2}

Protocolo de Observación de la Función de Explicación, PROFE

\begin{tabular}{|c|c|c|}
\hline CRITERIOS & \multicolumn{2}{|l|}{ CÓDIGOS } \\
\hline \multirow{6}{*}{$\begin{array}{l}\text { Organización } \\
\text { del contenido }\end{array}$} & Sintetizar & $\mathrm{SN}$ \\
\hline & Esquema & EQ \\
\hline & Conexión con contenidos anteriores & $\mathrm{CA}$ \\
\hline & Conexión con contenidos posteriores & $\mathrm{CP}$ \\
\hline & Explicación lineal & EX \\
\hline & Explicación con recursos & ER \\
\hline \multirow{8}{*}{$\begin{array}{c}\text { Relaciones } \\
\text { con los } \\
\text { contenidos }\end{array}$} & Uso de sinónimos & US \\
\hline & Analogías & $\mathrm{AN}$ \\
\hline & Generalización & GE \\
\hline & Repetición & $\mathrm{RP}$ \\
\hline & Definición & DF \\
\hline & Lectura & $\mathrm{LC}$ \\
\hline & Dictado & DC \\
\hline & Anécdotas & $\mathrm{AD}$ \\
\hline \multirow{3}{*}{$\begin{array}{l}\text { Contraste } \\
\text { estimular }\end{array}$} & Cambio de sonido & CS \\
\hline & Uso del humor & UH \\
\hline & Interacciones generales & IG \\
\hline \multirow{2}{*}{ Instrumental } & Inobservable & $\mathrm{Y}$ \\
\hline & Interrupción & IN \\
\hline
\end{tabular}

\section{Procedimiento}

En primer lugar, se grabaron en video ocho horas de docencia para cada profesor, escogiendo aquellas sesiones con mayor calidad de sonido e imagen. En segundo lugar, se comprobó la fiabilidad inter observadores y la homogeneidad de la última versión del instrumento, en su depuración definitiva, una vez creado el instrumento (Borges et al., 2016). Para asegurar una codificación eficiente, esto es, seleccionar tanto sesiones como tiempo dentro de la misma sin perder precisión, se realizó una prueba de optimización (Rodríguez-Dorta \& Borges, 2015; 2016), mediante la Teoría de la Generalizabilidad [TG] (Cronbach, Gleser, Nanda \& Rajaratnam, 1972), para la fase de desarrollo de la clase. Finalmente, se codificaron las conductas de los cinco profesores, para poder recoger todo el flujo de comportamientos, permitiendo establecer patrones comportamentales.

\section{Análisis de datos}

Para el análisis de fiabilidad se emplearon el índice Kappa de Cohen (Cohen, 1960) (analizado con el SPSS V.15), que estudia la concordancia entre los observadores, midiendo el grado de acuerdo entre los mismos y la Teoría de la Generalizabilidad [TG] (Cronbach et al., 1972), procedimiento de análisis que permite aislar las diversas fuentes de error de la medida, a la vez que ayuda a determinar de qué forma se pueden modificar los diversos factores, analizando su efecto en el coeficiente de generalizabilidad (Blanco \& Anguera, 2000). La TG ofrece un marco adecuado para buscar las estimaciones de fiabilidad y de márgenes de error, presentando un carácter globalizador que se adapta a las condiciones particulares de cada objeto de medida (Blanco, Castellano, \& Hernández Mendo, 2000; Blanco, et al., 2010). Su cálculo se realizó con el programa SGAT v. 1.0. (Ramos, Hernández, Pastrana \& Blanco, 2012), empleado igualmente para el análisis de optimización, el cual determina, si el número de docentes permite la generalización de resultados y el tiempo mínimo de codificación, así como, el número de sesiones necesarios para evitar perder precisión.

La conducta del profesor se examinó, a nivel de macronálisis (Sarriá, 2010) calculando como parámetro la frecuencia relativa, mientras que la determinación de patrones de comportamientos se hizo mediante el análisis secuencial, que sirve para detectar patrones de conducta (Sackett, 1987) y se basa en determinar si las secuencias de conductas tienen lugar con mayor probabilidad de lo que cabría esperar por azar. Para ello, se elige una conducta criterio, a partir de la cual se contabilizan las veces que las otras conductas la siguen en el siguiente lugar de orden, para el primer retardo, y con dos conductas, para el segundo, dándose una dependencia excitatoria o positiva: cuando $Z$ es mayor que 1.96 , o negativa, cuando $Z$ es menor de -1.96 . El retardo positivo 1 , permite establecer una asociación entre un determinado comportamiento y el comportamiento inmediatamente posterior, mientras que el retardo 2 establece la misma asociación pero con un segundo comportamiento posterior (Eide, Quera \& Finset, 2003). En el presente estudio se recogen ambos retardos. El análisis secuencial se llevó a cabo con el programa SDIS-GSEQ v. 5.1 (Bakeman \& Quera, 1996). Para determinar la cuantía de la asociación entre 
Ma África Borges del Rosal, Cristina Falcón Muñoz de Bustillo.

conductas, se ha calculado, mediante el mismo programa, el coeficiente $Q$ de Yule (Yule \& Kendal, 1937).

\section{Resultados}

Fiabilidad y homogeniedad de la última versión del instrumento

Para garantizar la calidad del dato, durante la creación y posterior depuración del instrumento se calculó la fiabilidad y homogeneidad del instrumento (ver tabla 3). La versión definitiva arroja resultados muy adecuados, como se puede comprobar. Posteriormente, en la codificación de los datos de este estudio, se realizó la fiabilidad inter observadores, obteniéndose valores que garantizan su fiabilidad (Kappa $=1$ y TG=0.98).

Tabla 3

Análisis de fiabilidad en la fase de depuración del instrumento

\begin{tabular}{lrcr}
\hline & Kappa & TG & Homogeneidad \\
\hline Versión 1 & 0.622 & 0.904 & 0.522 \\
Versión 2 & 0.886 & 0.963 & 0 \\
Versión 3 & 1 & 0.912 & 0 \\
\hline
\end{tabular}

Optimización de la muestra

Para determinar si el número de docentes empleados en la muestra permitía la generalizabilidad de resultados, se realizó un análisis de optimización mediante TG, optimizando el número de docentes, utilizando un diseño unifaceta cruzado, $\mathrm{CxP}$, siguiendo un Plan de Estimación de efectos aleatorios, donde la faceta de diferenciación son los códigos y la faceta a generalizar el número de docentes C/P. Los resultados obtenidos se presentan en la tabla 4.
Tabla 4

Plan de optimización del número de docentes $\mathrm{C} / \mathrm{P}$

\begin{tabular}{crrrrrr}
\hline Facetas & Niveles & Opt.1 & Opt.2 & Opt.3 & Opt.4 & Opt.5 \\
\hline $\mathrm{P}$ & $n=4$ & 5 & 6 & 7 & 8 & 9 \\
$\mathrm{C}$ & $n=35$ & 35 & 35 & 35 & 35 & 35 \\
\hline Coeficiente G relativo & 0.962 & $\mathbf{0 . 9 9 5}$ & 0.996 & 0.996 & 0.996 & 0.996 \\
Coeficiente G absoluto & 0.962 & $\mathbf{0 . 9 9 5}$ & 0.996 & 0.996 & 0.996 & 0.996 \\
\hline \multicolumn{4}{r}{ Nota: $\mathrm{P}=$} & Profesoras; C = Códigos
\end{tabular}

Optimización del tiempo a codificar

La determinación tanto del número de sesiones como del tiempo mínimo necesario de codificación, se realizó mediante TG, optimizándose en primer lugar el número de sesiones necesarias para cada docente, valiéndose de un diseño unifaceta cruzado, $\mathrm{CxS}$, siguiendo un Plan de Estimación de efectos aleatorios, donde la faceta de diferenciación son los códigos y la faceta a generalizar el número de sesiones, $\mathrm{C} /$ S.

A continuación, se optimizó el tiempo mínimo necesario correspondiente a la parte intermedia en cada uno de los docentes, utilizando el mismo diseño unifaceta cruzado, CxT y un Plan de Estimación de efectos aleatorios, donde la faceta de diferenciación fueron los códigos y la generalización del tramo de tiempo, así como la parte intermedia del desarrollo proveniente de la explicación docente, $\mathrm{C} / \mathrm{T}$. Las sesiones y tiempos que resultaron necesarias para cada docente se presentan en la tabla 5.

\section{Tabla 5}

Análisis de optimización de sesiones y tramos de tiempo

\begin{tabular}{|ccc|}
\hline Docente & Optimización Sesiones & Optimización Tramos \\
\hline Docente 1 & 2 Sesiones & 5 Tramos de 5 minutos \\
Docente 2 & 2 Sesiones & TG $=0.99$ \\
& $\mathrm{TG}=0.95$ & 2 Tramos de 5 minutos \\
Docente 3 & 2 Sesiones & TG $=0.93$ \\
& $\mathrm{TG}=0.99$ & $\mathrm{~T}$ ramos de 5 minutos \\
Docente 4 & 2 Sesiones & 2 Tramos de 5 minutos \\
& $\mathrm{TG}=0.99$ & $\mathrm{TG}=0.946$ \\
Docente 5 & $2 \mathrm{Sesiones}$ & 5 Tramos de 5 minutos \\
& $\mathrm{TG}=0.96$ & $\mathrm{TG}=0.919$ \\
\hline
\end{tabular}

Nota: $\mathrm{TG}=$ Teoría de la Generalizabilidad 
Dada la naturaleza del contexto objeto de estudio de la presente investigación, restringir los análisis al número de sesiones obtenidas en el proceso de optimización parece limitado (Rodríguez-Dorta \& Borges, 2016). Por ello, según el principio de saturación, se aumentaron en una el número de sesiones a analizar, para así obtener información más fidedigna acerca del comportamiento del profesor dentro del contexto educativo.

Análisis de los comportamientos codificados del profesorado

Las frecuencias relativas de aparición de las conductas durante el desarrollo de la clase, se muestran en la tabla número 6 , donde se incluye el código siempre que al menos uno de los docentes haya usado la estrategia analizada. Las conductas más frecuentes son 'Explicación lineal' de los contenidos y 'Explicación con recursos', lo cual resulta coherente, porque es esperable que la conducta más frecuente del docente, para impartir contenidos, sea explicar, con o sin recursos. No obstante, se observa que todo el profesorado participante en el estudio hace uso de una u otra estrategia, siendo las más frecuentes 'Conexión con contenidos anteriores' y el 'Uso de ejemplos', mientras que 'Uso del humor', 'Anécdotas', 'Conexión con contenidos posteriores, Definición', 'Generalización', 'Analogías', 'Uso de sinónimos' tienen una frecuencia muy baja. Otras estrategias, como 'Lectura', 'Repetición' y 'Síntesis' son utilizadas solo por uno de los docentes. No aparecen el resto de códigos que forman el instrumento ('Esquema', 'Dictado, Cambio de sonido', 'Interrupción', 'Interacciones generales' y 'Conductas inobservables').
Tabla 6

Análisis de la frecuencia relativa. Instrumento PROFE. Intermedio

\begin{tabular}{|cccccc|}
\hline & Docente 1 & Docente 2 & Docente 3 & Docente 4 & Docente 5 \\
\hline $\begin{array}{c}\text { Sintesis } \\
\text { Conexión con }\end{array}$ & $0 \%$ & $0 \%$ & $0 \%$ & $5 \%$ & $0 \%$ \\
contenidos anteriores & $1 \%$ & $3 \%$ & $9 \%$ & $7 \%$ & $2 \%$ \\
$\begin{array}{c}\text { Conexión con } \\
\text { contenidos posteriores }\end{array}$ & $2 \%$ & $3 \%$ & $0 \%$ & $2 \%$ & $1 \%$ \\
Explicación lineal & $35 \%$ & $45 \%$ & $44 \%$ & $32 \%$ & $48 \%$ \\
$\quad \begin{array}{c}\text { Explicación con } \\
\text { recursos }\end{array}$ & $33 \%$ & $17 \%$ & $29 \%$ & $45 \%$ & $24 \%$ \\
$\quad$ Ejemplos & $4 \%$ & $13 \%$ & $11 \%$ & $1 \%$ & $6 \%$ \\
Sinónimos & $0 \%$ & $0 \%$ & $0 \%$ & $2 \%$ & $1 \%$ \\
$\quad$ Analogias & $3 \%$ & $0 \%$ & $1 \%$ & $2 \%$ & $4 \%$ \\
Generalizaciones & $2 \%$ & $2 \%$ & $1 \%$ & $1 \%$ & $3 \%$ \\
Repetición & $0 \%$ & $0 \%$ & $0 \%$ & $1 \%$ & $0 \%$ \\
Definición & $1 \%$ & $5 \%$ & $2 \%$ & $3 \%$ & $6 \%$ \\
$\quad$ Lectura & $15 \%$ & $0 \%$ & $0 \%$ & $0 \%$ & $0 \%$ \\
$\quad$ Anécdotas & $4 \%$ & $9 \%$ & $1 \%$ & $0 \%$ & $4 \%$ \\
Uso del humor & $1 \%$ & $2 \%$ & $2 \%$ & $0 \%$ & $2 \%$ \\
Interacciones generales & $0 \%$ & $1 \%$ & $1 \%$ & $0 \%$ & $0 \%$ \\
\hline
\end{tabular}

El análisis secuencial se llevó a cabo de forma separada para cada docente, presentándose en la tabla 7 los patrones significativos, entre paréntesis el valor del residual y en la última columna el estadístico $Q$ de Yule (Yule \& Kendal, 1937), que determina la cuantía de la relación. En el primer retardo, la 'Explicación lineal', tomada como antecedente, seguida de 'Explicación con recursos', es el patrón más frecuente, pues resulta significativo en todo el profesorado estudiado. Eso pone de manifiesto que éste introduce contenidos y se apoya en el recurso multimedia para complementar su exposición. Cuando la conducta antecedente es 'Explicación lineal', se producen patrones significativos, pero en este caso son específicos de cada docente. 
Tabla 7

Análisis secuencial con dos retardos

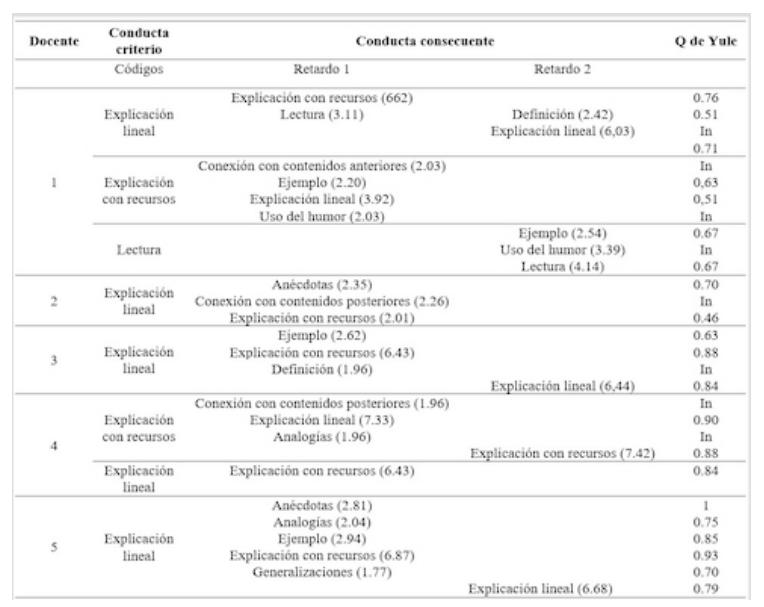

Finalmente, otra conducta que lleva a patrones significativos en dos de los docentes es la 'Explicación con recursos', seguida de comportamientos diferentes en cada caso. Aparecen patrones comportamentales significativos en segundo retardo en todos los docentes, excepto en el segundo. El patrón más frecuente, es aquel dónde la conducta criterio, 'Explicación con recursos', va seguida, en segundo retardo por esa misma conducta. Ello significa que, el docente explica, hace uso de alguna estrategia para reforzar la transmisión de contenidos, continuando con la explicación. Otros patrones, que aparecen en segundo retardo son 'Definición', 'Uso de Ejemplos', 'Uso de recursos multimedia', 'Uso del humor o Lectura'. Todo ello señala que la impartición de contenidos por parte de los docentes estudiados no es plana, sino que hacen uso de diferentes procedimientos para facilitar la comprensión de contenidos por parte de su alumnado.

Para comprobar el grado de asociación entre los patrones se ha calculó el estadístico $Q$ de Yule (Yule \& Kendal, 1937), que se muestra en la columna de la derecha de la tabla 5, observándose asociaciones medias o altas, pues las relaciones obtenidas son superiores a 0.50 . No obstante, cuando alguna de las casillas de la tabla de contingencia es nula, no es posible calcular la $Q$ de Yule (Yule \& Kendal, 1937), por lo que resulta indefinida (In).

\section{Conclusión}

La evaluación de la conducta del profesorado, es un tema de absoluta relevancia dentro del aseguramiento de la calidad universitaria. Por esa razón, el desarrollo de investigaciones en las que se evalúe su comportamiento durante la exposición de contenidos es fundamental, en la medida que permite determinar las estrategias seguidas.

El instrumento, ha demostrado calidad del dato, dados los buenos resultados obtenidos en la creación del instrumento, tanto en fiabilidad entre observadores como en homogeneidad, que garantiza la independencia de los códigos. La fiabilidad, además, se garantiza en la codificación posterior, lo que permite confirmar la calidad del dato usando este instrumento en la investigación.

Los resultados obtenidos en el presente estudio, muestran que cada uno de los y las docentes, hace un uso adecuado de las estrategias instruccionales. El código con mayor frecuencia de uso es 'Explicación lineal', que configura la exposición de contenidos, seguida por 'Explicación con recursos'. Cabe destacar que todo el profesorado estudiado, en mayor o menor medida, hace uso de unas u otras estrategias, sean de organización del contenido, de relación con el contenido o de contraste estimular.

Es coherente que las estrategias más frecuentemente utilizadas, sean las relacionadas con exponer contenidos, operacionalizada en este estudio con el código 'Exposición Lineal', pues el objetivo del profesorado es conseguir una transmisión eficaz de los mismos, pero también es necesario aplicar contrastes estimulares, ya que esta técnica motivacional, basada en el principio gestáltico, contrasta figura- fondo y situada en el primer momento de la motivación, es clave para suscitar la atención del alumnado (Hernández, 1991). Entrenar al profesorado en la correcta aplicación de este contraste, podría ser de gran utilidad, pues se lograría una transmisión más eficaz de los contenidos. En la presente investigación, el contraste estimular más frecuentemente utilizado es el 'Uso del humor'. 
Los resultados obtenidos en el análisis secuencial, permiten descubrir patrones interesantes, que aportan mucha información y ayudan a una mejor comprensión del desarrollo de las clases. El profesorado, explica los contenidos correspondientes apoyándose en diferentes materiales multimedia. El siglo XXI y la llegada de las nuevas tecnologías, han permitido incorporar nuevas formas de difundir contenidos dentro del contexto educativo, siendo una de sus ventajas el enriquecer y aumentar el atractivo de las clases. Estos nuevos procedimientos también han irrumpido con fuerza en el contexto universitario, como se puede comprobar asistiendo a cualquier clase de cualquier universidad española, que se han puesto de manifiesto en esta investigación. Tal y como señalan Fombona y Pascual (2011), con el desarrollo de las TIC, éstas se han incorporado con gran pujanza en los sistemas de educación superior, provocando una modificación de sus estructuras, que incluyen las competencias digitales como elementos esenciales para desenvolverse en nuestra sociedad y generar un conocimiento elaborado.

La explicación, tanto lineal como con recursos, va acompañada del uso de ejemplos, estrategia que resulta de gran utilidad, pues permite la clarificación y comprensión de los contenidos al conectar con las experiencias concretas del alumno (Jiménez \& Hernández-Jorge, 2001). El profesor, además enlaza la explicación de los contenidos actuales con contenidos anteriores o posteriores, lo cual permite dotar de una mayor coherencia al proceso. Puede aparecer seguida de anécdotas o uso del humor, que como se ha descrito anteriormente, permite suscitar la atención, o bien de estrategias correspondientes al uso de definiciones, generalizaciones y lectura.

Dado que la calidad de la Educación Superior es un eje fundamental en el proceso de Bolonia, y a su vez, dentro de ella la aportación del profesorado es esencial, conocer cómo se comporta dentro del escenario instruccional es un aspecto de gran importancia. Utilizar en su evaluación instrumentos observacionales, posibilita la valoración del docente a la vez que, debido al nivel del detalle de la conducta del profesorado la cual permite alcanzar, favorecer la opción de analizar y detectar las limitaciones que pueda tener un docente o una docente concretos. Por tanto, estableciendo recomendaciones de mejora que contribuyan a mejorar la calidad de su docencia y, en definitiva, la calidad del proceso educativo. La evaluación del profesorado, es esencial para garantizar la calidad de la enseñanza, y la metodología observacional en general y el instrumento que aquí se presenta, en particular, favorece la evaluación en el entorno real del desempeño docente, dentro del aula, mientras imparte docencia.

Este estudio ha contado con un limitado número de docentes participantes, lo que constituye su principal debilidad, si bien, como se ha dicho, los resultados de optimización permiten su generalizabilidad. Además, por una parte, el acercamiento en profundidad que supone usar la metodología observacional, no resulta especialmente apropiado para muestras numerosas. Por otra parte, el objetivo de este estudio no estaba orientado a la descripción de muestras generales de comportamiento, sino en mostrar la utilidad que puede tener un instrumento como el aquí presentado de cara a la evaluación del profesorado, y, por ende, a determinar su calidad como docente.

En futuras investigaciones sería muy interesante seguir analizando la conducta del profesorado, contando para ello con un mayor número de docentes y la colaboración de otras universidades a nivel nacional e internacional, para garantizar la aplicabilidad del instrumento y su validez cruzada.

\section{Agradecimientos}

A los diferentes profesores que han formado parte de esta investigación.

\section{Referencias}

Aciego de Mendoza, R., Domínguez, R. \& Martín, E. (2002). Metodología docente utilizada por el profesorado universitario. 
Ma África Borges del Rosal, Cristina Falcón Muñoz de Bustillo.

Cultura y Educación, 14(2), 177-186. https: //doi.org/10.1174/113564002760041569.

Anguera, M.T., Blanco, A., \& Losada, J.L. (2001). Diseños observacionales, cuestión clave en el proceso de la metodología observacional. Metodología de las Ciencias del Comportamiento, 3(2), 135-160.

Anguera, M. T., \& Hernández-Mendo, A. (2013). La metodología observacional en el ámbito del deporte. Ebalonmano.com: Revista de Ciencias del Deporte, 9(3), 135-160. Recuperado de http://www.e-balonmano.com/ojs/index .php/revista/article/view/139/pdf 20

Ausubel, D. P. (1968). Educational Psichology: A congnitive view. New York: Holt, Rinehart \& Winston.

Bakeman, R. \& Quera, V. (1996). Análisis de la interacción. Análisis Secuencial con SDIS y GSEQ. Madrid: RA-MA S.A. Editorial y Publicaciones.

Blanco, A. \& Anguera, M.T. (2000). Evaluación de la calidad en el registro del comportamiento: Aplicación a deportes de equipo. En E. Oñate, F. GarcíaSicilia \& L. Ramallo (Eds.), Métodos numéricos en Ciencias Sociales (pp. 30-48). Barcelona: Centro Internacional de Métodos Numéricos en Ingeniería (CIMNE).

Blanco, A., Castellano, J., \& Hernández Mendo, A. (2000). Generalizabilidad de las observaciones en la acción del juego en el fútbol. Psicothema, 12(2), 81-86. Recuperado de http://psicologia.del.deport e.uma.es/archivos/3generalizabilidad.pdf

Blanco, A., Sastre, S., \& Escolano, E. (2010). Desarrollo ejecutivo temprano y Teoría de la Generalizabilidad: bebés típicos y prematuros. Psicothema, 22(2), 221-226. Recuperado de https:/www.researchgate.net/publicatio n/43602936_Desarrollo_ejecutivo_tempra no_y_Teoria_de_la_Generalizabilidad_beb es_tipicos_y_prematuros

Borges, A. \& Díaz, M. (2013). Evaluación de la Docencia Universitaria y su profesorado. La calidad del proceso de enseñanza y aprendizaje. Berlín: Editorial Academia Española.

Borges, A., Falcón, C., \& Díaz, M. (2016). Creation of an Observational Instrument to Operationalize the Transmission of Contents by University Teachers. International Journal of Social Science Studies, 4(7), 82-89. https://doi.org/10.11114/ijsss.v $4 \mathrm{i} 7.1596$

Cameron, C. E., Connor, C. M., \& Morrison, F. J. (2005). Effects of variation in teacher organization on classroom functioning. Journal of School Psychology, 43(1), 61-85. h ttps://doi.org/10.1016/j.jsp.2004.12.002

Cohen, J. (1960). A coefficient of agreement for nominal scales. Educational and Psychological Measurement, XX(1), 37-46. Recuperado de ftp://gis.msl.mt.gov/Maxell/Models/ Predictive Modeling for DSS Lincoln NE 121510/Modoling_Literature/Cohen_1960.pdf

Cronbach, L.J., Gleser, G.C., Nanda, H., \& Rajaratnam, N. (1972). The dependability of behavioral measurements: theory of generalizability for scores and profiles. Nueva York: John Wiley and Sons.

Díaz, M. (2014). Protocolo de Observación de Funciones Docentes en Universidad: Un instrumento para la evaluación de la conducta del profesorado universitario. (Tesis Doctoral). Soportes Audiovisuales e Informáticos Universidad de La Laguna, España. Recuperado de https://riull.ull.es/x mlui/handle/915/94

Díaz, M. C., Borges, A., Valadez-Sierra, D., \& Zambrano-Guzmán, R. (2014). Evaluación docente del profesor universitario de la carrera de Psicología en la Universidad de Guadalajara. Revista de Educación y Desarrollo, 28, 5-14. Recuperado de http://www.cucs.udg.mx/revistas/edu_d esarrollo/anteriores/28/028_Diaz.pdf

Díaz, M., Borges, A., Valadez, M.D., \& Zambrano, R. (2015a). Valoración de buenas prácticas docentes a través de observación sistemática. Universitas Psychologica, 14(3), 913-922. https://doi.org /10.11144/Javeriana.upsy14-3.vbpd 
Díaz, M., Borges, A., Valadez, M. D., \& Zambrano, R. (2015b). Evaluation of the Teaching Performance of University Lecturers: Comparison between Mexico and Spain. Journal of Curriculum and Teaching, 4(2), 53-61. https://doi.org/10.54 30/jct.v4n2p53

Díaz, C., Borges, A. \& Valadez, M.D. (2013). Evaluación de la conducta del docente, una propuesta para el diagnóstico del Burnout. En N. Rubalcaba, L.E. Oliveros, R. Flores, M. G. Covarrubias y J.J. Gutiérrez (coord.), Aportes Interdisciplinarios en el ejercicio profesional de la Salud Mental, Vol.II (pp.166-177). Guadalajara: Universidad de Guadalajara, Centro Universitario de Ciencias de la Salud.

Eide, H., Quera, V., \& Finset, A. (2003). Exploring rare patient behaviour with sequential analysis: an illustration. Epidemiology and Psychiatric Sciences, 12 (2), 109-114. https://doi.org/10.1017/S1121189 X00006175

Falcón, C. (2015). Protocolo de Observación de la Función de Explicación (PROFE): Un instrumento para operacionalizar la transmisión de contenidos por parte del profesorado. (Tesis de xxx). Universidad de La Laguna. Tenerife.

Fombona, J. \& Pascual, M.A. (2011). Las tecnologías de la información y la comunicación en la docencia universitaria. Educación XXI, 14(2), 79-110. https://doi.o $\mathrm{rg} / 10.5944 /$ educxx1.14.2.246

Galán, A., González-Galán, M.A., \& RodríguezPatrón, P. (2014). La evaluación del profesorado universitario en España. Sistema nacional y divergencias territoriales. Revista de Educación, 366, 136-164. https://doi.org/10.4438/1988-592 X-RE-2014-366-279

García, L.A. (1998) Psicología instruccional $e$ intervención para la mejora cognitiva. Memoria de Cátedra: Universidad de La Laguna.

Good, T. L., Mulryan, C., \& McCaslin, M. (2006). Grouping for instruction in mathematics: A call for programmatic research on small-group processes. En D. Grouws (Ed.), Handbook of research on mathematics teaching and learning (pp. 165196). Nueva York: Macmillan.

Hernández, P. (1991). Psicología de la Educación: corrientes actuales y teorías aplicadas. México: Trillas.

Hernández, P. (1997). Construyendo el constructivismo: Criterios para su fundamentación y su aplicación instruccional. En M. J. Rodrigo y J. Arnay, Constructivismo del conocimiento escolar (pp. 285-312). Barcelona: Paidós.

Hernández, P. \& García, L. (1995). Análisis y organización de los contenidos. En P. Hernández (comp) Diseñar y enseñar: Teoría y técnicas de la programación y del proyecto docente. Madrid: Narcea.

Hernández-Jorge, C. (2005). Habilidades de comunicación para profesionales. Tenerife: ARTE Comunicación Visual.

Jiménez, H. \& Hernández-Jorge, C. (2001). ¿Cómo comunicarnos eficazmente en la enseñanza? La comunicabilidad didáctica. En A. Rodríguez (coord.), Psicología de la Instrucción para enseñantes (pp. 183-197). Las Palmas de Gran Canaria: Instituto Psicosocial Manuel Alemán.

Ley Orgánica 6/2001. Boletín Oficial del Estado, no 307, España, 24 de diciembre de 2001.

Martínez, J.F. (2013). Combinación de mediciones de la práctica y el desempeño docente: consideraciones técnicas y conceptuales para la evaluación docente. Pensamiento educativo. Revista de Investigación Educacional Latinoamericana, 50(1), 4-20. https://doi.org/10.7764/PEL.5 0.1 .2013 .2

Mateo, J. \&Martínez, F. (2008). La evaluación alternativa de los aprendizajes. Barcelona: Octaedro. Recuperado de http://www.ub.edu/ice/sites/default/files/ docs/qdu/3cuaderno.pdf

Mayorga, M. J., \& López, A. (2005). Observar para evaluar al profesorado: una experiencia práctica. Revista de Educación, 7, 167-181. Recuperado de http://rabida.uhu.es/dspace/bitstream/h 
andle/10272/1955/b15166429.pdf?sequenc $\mathrm{e}=1$

Pianta, R. C. \& Hamre, B. K. (2009). Conceptualization, measurement, and improvement of classroom processes: Standardized observation can leverage capacity. Educational Researcher, 38(2), 109-119. https://doi.org/10.3102/0013189 X09332374

Ramos, FJ., Hernández-Mendo, A., Pastrana, J.L., \& Blanco, A. (2012). SGAT: Software para la Aplicación de la Teoría de la Generalizabilidad v 1.0. (). Ingeniería Técnica en Informática de Gestión de la Escuela Técnica Superior de Ingeniería Informática. Universidad de Málaga.

Rodríguez-Dorta, M. y Borges, A. (2015). Optimización del tiempo y número de sesiones para la observación de las funciones docentes en educación primaria y especial. En A. Borges y S. Hess (coord.) XIII Congreso de Metodología de las Ciencias Sociales y de la Salud (pp. 85-96). La Laguna: Servicio de Publicaciones de la Universidad de La Laguna.

Rodríguez-Dorta, M. \& Borges, A. (2016). Optimización y eficiencia en el análisis de datos en observacional. Revista Electrónica de Metodología Aplicada, 21 (1), 1-15. https: //doi.org/10.17811/rema.21.1.2016.1-15

Rodríguez-Dorta, M. \& Borges, Á. (2017). Behavioral patterns in Special Education. Good teaching practices. Frontiers in Psychology, 8, 631. https://10.3389/fpsyg.20 17.00631

Rodríguez-Naveiras, E. \& Borges, A. (2015). Optimización de las actividades de un programa extraescolar de altas capacidades para la calidad del dato en la evaluación de la conducta del monitor. En A. Borges y S. Hess (coord.) XIII Congreso de Metodología de las Ciencias Sociales y de la Salud (pp. 54-63). La Laguna: Servicio de Publicaciones de la Universidad de La Laguna.

Román, M. \& Murillo. J. F. (2013). Evaluación en el campo educativo: del sentido a la práctica [Editorial]. Revista Iberoamericana de Evaluación Educativa, 6(1), 7-12. Recuperado de https://repositorio.uam.es/b itstream/handle/10486/661322/evaluacion roman_RIEE_2013.pdf?sequence $=1$ \&is $\overline{\text { Allowe }} \overline{\mathrm{d}}=\mathrm{y}$

Sackett, G.P. (1987). Analysis of sequential social interaction data: Some issues, recent developments, and a causal inference model. En J.D. Osofsky (Ed). Handbook of infant development (pp. 855-878). Nueva York: Wiley.

Sarriá, E. (2010). La observación. En S. Fontes, C. García-Gallego, L. Quintanilla, R. Rodríguez, P. Rubio \& E. Sarriá, Fundamentos de investigación en Psicología (pp. 313 - 353). Madrid: UNED.

Yule, G.V. \& Kendall, M.G. (1937). An introduction to the theory of statistics. London: Griffin.

\section{Notas}

* Artículo de investigación. 